\title{
Driving, Conservation, and Absorbing States in Sandpiles
}

\author{
Alessandro Vespignani, ${ }^{1}$ Ronald Dickman, ${ }^{2}$ Miguel A. Muñoz, ${ }^{1,3}$ and Stefano Zapperi ${ }^{4}$ \\ ${ }^{1}$ The Abdus Salam International Centre for Theoretical Physics (ICTP), P.O. Box 586, 34100 Trieste, Italy \\ ${ }^{2}$ Departamento de Física, Universidade Federal de Santa Catarina, Campus Universitário, Trindade, \\ CEP 88040-900, Florianópolis - SC, Brazil \\ ${ }^{3}$ Dipartimento di Fisica e unità INFM, Università di Roma "La Sapienza," Piazzale A. Moro 2, I-00185 Roma, Italy \\ ${ }^{4}$ PMMH-ESPCI, 10, rue Vauquelin, 75231 Paris CEDEX 05, France \\ (Received 18 June 1998; revised manuscript received 5 November 1998)
}

We use a phenomenological field theory, reflecting the symmetries and conservation laws of sandpiles, to compare the driven dissipative sandpile, widely studied in the context of self-organized criticality, with the corresponding fixed-energy model. The latter displays an absorbing-state phase transition with upper critical dimension $d_{c}=4$. We show that the driven model exhibits a fundamentally different approach to the critical point, and compute a subset of critical exponents. We present numerical simulations in support of our theoretical predictions. [S0031-9007(98)07996-4]

PACS numbers: 64.60.Lx, 05.40. $+\mathrm{j}$, 05.70.Ln, 46.10.+z

A wide variety of nonequilibrium systems display transitions between "active" and "absorbing" states: examples are epidemic processes [1], catalysis [2], directed percolation (DP) [3], and the depinning of interfaces in quenched disorder [4]. When driven continuously, such systems may exhibit stick-slip instabilities, or broadly distributed avalanches, commonly associated with self-organized criticality (SOC) $[5,6]$.

SOC sandpiles [5] possess an infinite number of absorbing configurations (i.e., from which the system cannot escape), and are placed, by definition, at the critical point in a two-dimensional parameter space $[7,8]$ resembling that of directed percolation (DP) [3] or contact processes [9-11].

Under an external drive (i.e., input of particles at rate $h$ ), the system jumps among absorbing configurations via avalanchelike rearrangements. Close to the absorbingstate phase transition, a slow drive induces avalanches whose size distribution decays as a power law-the hallmark of SOC. What distinguishes the sandpile from other models with absorbing states is a conservation law: avalanche dynamics conserves the number of grains of "sand," and the order parameter is coupled to this conserved field [8].

In this Letter we use a phenomenological field theory of sandpiles to show how conservation alters the phase transition. The critical behavior for $h \longrightarrow 0$ (the SOC limit) differs from that for $h \equiv 0$. In particular, when driving and dissipation are absent, the sandpile shows an absorbing-state phase transition (with $d_{c}=4$ ). Our approach clarifies the effect of driving on dynamic phase transitions, and resolves several long-standing issues regarding sandpiles, such as the upper critical dimension, the effect of conservation on critical exponents, and universality classes [12-16]. We perform extensive simulations to check our theoretical predictions.

In sandpiles [5], each site $i$ of a $d$-dimensional lattice bears an integer variable $z_{i} \geq 0$, which we call energy.
When a site reaches or exceeds a threshold $z_{c}$ it topples: $z_{i} \longrightarrow z_{i}-z_{c}$, and $z_{j} \longrightarrow z_{j}+1$ at each of the $g$ nearest neighbors of $i$. Energy is fed into the system at rate $h$, and is dissipated at rate $\epsilon$ during toppling [7]. At each time step, each site has a probability $\propto h$ to receive an energy grain; in each toppling, a grain is lost with probability $\propto \epsilon$. These rules generalize the original Bak, Tang, and Wiesenfeld (BTW) sandpile automaton [5], which is recovered in the limit $h \longrightarrow 0$ and $\epsilon \longrightarrow 0$ $[7,8]$. While the BTW model restricts dissipation to the boundaries, we focus on (conceptually simpler) bulk dissipation; most conclusions apply to the boundary dissipation case as well. We also consider the Manna sandpile [17], in which $z_{c}=2$ and two neighboring sites are chosen at random to receive energy.

In the slow-driving limit $\left(h \rightarrow 0^{+}\right)$each energy addition is followed by an avalanche of $s$ topplings; the avalanche distribution has the scaling form $P(s)=$ $s^{-\tau} G\left(s / s_{c}\right)$, where the cutoff scales as $s_{c} \sim \xi^{D}$. The correlation length $\xi$ scales with dissipation as $\xi \sim \epsilon^{-\nu}$, and is related to the characteristic avalanche duration $t_{c} \sim \xi^{z}$.

The order parameter is $\rho_{a}$, the density of active sites (i.e., whose height $z \geq z_{c}$ ) [7,8]; if $\rho_{a}=0$ the system has reached an absorbing configuration. In a coarsegrained description, we study the dynamics of a local order-parameter field $\rho_{a}(\mathbf{x}, t)$, bearing in mind that the energy density $\zeta(\mathbf{x}, t)$ is (for $\epsilon=h=0$ ), a conserved field. Variations of the local energy density are due to: (i) the external field, $h$; (ii) dissipation attending toppling: $-\epsilon \rho_{a}$; (iii) a diffusionlike contribution: $(1-\epsilon) \nabla^{2} \rho_{a}$. The latter arises because a gradient in $\rho_{a}$ leads to a current: the excess in the mean number of particles arriving at $\mathbf{x}$ from the left, over those arriving from the right, is $j_{x}(\mathbf{x}, t)=-(1-\epsilon) \partial_{x} \rho_{a}$. The net inflow of particles at $\mathbf{x}$ is therefore $-\nabla \cdot \mathbf{j}=(1-\epsilon) \nabla^{2} \rho_{a}$. Defining an energy diffusion constant $D_{\zeta} \propto 1-\epsilon$, we write the continuity equation for the energy density, 


$$
\begin{aligned}
\frac{\partial \zeta(\mathbf{x}, t)}{\partial t}= & D_{\zeta} \nabla^{2} \rho_{a}(\mathbf{x}, t)-\epsilon \rho_{a}(\mathbf{x}, t) \\
& +h(\mathbf{x}, t)+\eta_{\zeta}(\mathbf{x}, t),
\end{aligned}
$$

where the driving field $h(\mathbf{x}, t)=\bar{h}+\eta_{h}(\mathbf{x}, t)$, with $\bar{h}$ a nonfluctuating term and $\eta_{h}$ zero-mean, uncorrelated Gaussian noise. The last term is dynamically generated Reggeon-field-theory-like (RFT) noise [18] $\eta_{\zeta}(\mathbf{x}, t) \sim$ $\sqrt{\rho_{a}(\mathbf{x}, t)} \eta(\mathbf{x}, t)$, with $\eta$ uncorrelated Gaussian noise. This term vanishes, as it must, in the absorbing state, $\rho_{a}=0$.

The equation for the order-parameter field is readily obtained [8] by extending the mean-field theory (MFT) of Ref. [7]. With $\rho_{c}(\mathbf{x}, t)$ the local density of "critical" sites (i.e., with height $z_{c}-1$ ), we have

$$
\begin{aligned}
\frac{\partial \rho_{a}(\mathbf{x}, t)}{\partial t}= & D_{a} \nabla^{2} \rho_{a}(\mathbf{x}, t)-\rho_{a}(\mathbf{x}, t) \\
& +(g-\epsilon) \rho_{a}(\mathbf{x}, t) \rho_{c}(\mathbf{x}, t)+h \rho_{c}(\mathbf{x}, t) \\
& +\eta_{a}(\mathbf{x}, t),
\end{aligned}
$$

where $\eta_{a}(\mathbf{x}, t)$ is a RFT-like noise whose amplitude is proportional to $\sqrt{\rho_{a}(\mathbf{x}, t)}$. The first two terms represent toppling [19]; the terms $\propto \rho_{c}$ represent critical sites becoming active upon receiving energy, whether from the external drive, or from toppling neighbors.

In the stationary state, we can avoid ensnarement in an infinite hierarchy of equations [8], by eliminating $\rho_{c}$ in favor of $\zeta$ and $\rho_{a}$. In the Manna model, we simply invoke normalization: $\rho_{c}=\zeta-z_{a} \rho_{a}$, where $z_{a} \geq 2$ is the mean height of active sites. For BTW we use the phenomenological ansatz: $\rho_{c}(\mathbf{x}, t)=[1-$ $\left.\rho_{a}(\mathbf{x}, t)\right] f[\zeta(\mathbf{x}, t)]$. That is, the fraction $f$ of nonactive sites that are critical can be expressed as a singlevalued function of the local energy density. In the slowly driven stationary state, $\zeta \simeq \zeta_{s}$ and $f \simeq \rho_{c}^{\infty}$, where $\zeta_{s}$ and $\rho_{c}^{\infty}$ are the stationary average values of the energy and the critical-site density. We expand $f(\zeta)=\rho_{c}^{\infty}+$ $A \Delta \zeta+\ldots$, where $\Delta \zeta \equiv \zeta(\mathbf{x}, t)-\zeta_{s}$, and $A>0$. We test the validity of these assumptions by simulating the two-dimensional BTW model on a lattice of $80 \times 80$ sites, at $\zeta=\zeta_{s}=2.125$. To determine $f(\zeta)$, we measure the average energy, and active- and critical-site densities in cells of $10 \times 10$ sites. The conditional probability density $P(f \mid \zeta)$ is unimodal and peaked (see Fig. 1); the mean increases linearly with $\zeta$, indicating that $\rho_{c}$ is well-approximated by $\left(1-\rho_{a}\right) f(\zeta)$, with $f$ linear in the neighborhood of $\zeta_{s}$.

Equations (1) and (2), describing the coarse-grained dynamics of sandpiles, resemble the field theory for the pair contact process (PCP) [11], another model with infinitely many absorbing states. As in the PCP, when $h=0$ all configurations $\zeta(\mathbf{x})$ consistent with $\rho_{a} \equiv 0$ are absorbing, and the order parameter is coupled to a non-order-parameter field playing the role of an effective creation rate. The essential difference in the sandpile is that the field $\zeta$ is conserved. In the following, we

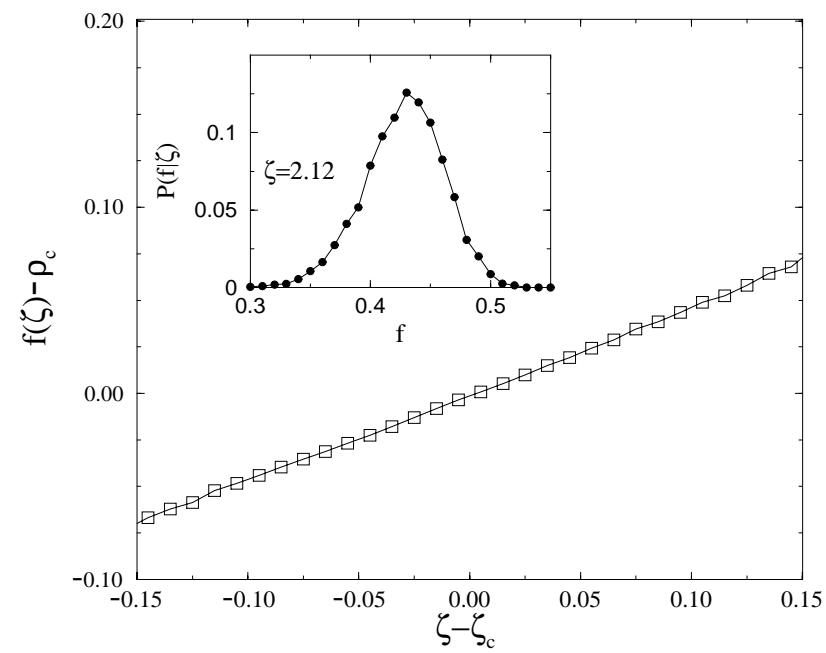

FIG. 1. Fraction of critical nonactive sites, $f$ (shifted by $\rho_{c}$ ) versus $\zeta-\zeta_{c}$ for the $\mathrm{BTW}$ in $d=2$. Inset: Conditional probability density at $\zeta=2.12$ for the same case.

consider separately the cases of slow driving $\left(h \longrightarrow 0^{+}\right)$, corresponding to the SOC sandpile, and of fixed energy: $h=0$ and $\epsilon=0$.

(i) Driven sandpile. - The system attains its stationary state by the very slow addition of energy. In this limit $\left(h \longrightarrow 0^{+}\right)$, a complete time scale separation, between toppling, on one hand, and addition and dissipation, on the other, sets in [6-8]. In the stationary state, energy balance forces a subset of the critical exponents to take their mean-field (MF) values in any spatial dimension $d$, as we now show. Integrating Eq. (1) over space and averaging over the noise yields $\left\langle\rho_{a}\right\rangle \equiv V^{-1} \int d^{d} x\left\langle\rho_{a}(\mathbf{x}, t)\right\rangle=h / \epsilon$ [7], which implies that the zero-field susceptibility $\chi \equiv$ $\partial \rho_{a} / \partial h$, diverges as $\epsilon^{-\gamma}$ with $\gamma=1$. Taking the functional derivative of Eq. (1) with respect to $h\left(\mathbf{x}^{\prime}\right)$, and averaging over the noise, we obtain an equation for the static response function $\chi_{\epsilon}\left(\mathbf{x}-\mathbf{x}^{\prime}\right)=\left\langle\delta \rho_{a}(\mathbf{x}) / \delta h\left(\mathbf{x}^{\prime}\right)\right\rangle$ :

$$
-D_{\zeta} \nabla^{2} \chi_{\epsilon}\left(\mathbf{x}-\mathbf{x}^{\prime}\right)+\epsilon \chi_{\epsilon}\left(\mathbf{x}-\mathbf{x}^{\prime}\right)=\delta\left(\mathbf{x}-\mathbf{x}^{\prime}\right),
$$

which yields, for large $\mathrm{r}, \chi_{\epsilon}(r) \propto r^{2-d} e^{-r / \xi}$, where the correlation length $\xi \sim \epsilon^{-\nu}$, with $\nu=1 / 2$. These results depend solely upon stationarity and translation invariance [20]. Although the exponent values coincide with the MF ones, they have not been obtained by MF arguments, and are valid beyond MFT, as confirmed by simulations in $2 \leq d \leq 6$ [14].

While the remaining exponents are in principle also determined by Eqs. (1) and (2), a full analysis, involving the double limit $t \longrightarrow \infty, h \longrightarrow 0^{+}$(the order cannot be interchanged), promises to be a knotty problem. The critical properties emerge as $\epsilon \longrightarrow 0$, which must be taken subsequent to the above limits, since a stationary state demands $\epsilon>h$. The upper critical dimension, however, can be found by power-counting analysis. The evolution of a localized perturbation $\rho_{a}$ around the slowly driven 
stationary state $(h / \epsilon=0)$ is given by

$\begin{aligned} \frac{\partial \rho_{a}(\mathbf{x}, t)}{\partial t}= & D_{a} \nabla^{2} \rho_{a}(\mathbf{x}, t)-r \rho_{a}(\mathbf{x}, t) \\ & +\mu \Delta \zeta(\mathbf{x}, t) \rho_{a}(\mathbf{x}, t)-u \rho_{a}^{2}(\mathbf{x}, t)+\eta_{a}(\mathbf{x}, t),\end{aligned}$

where $r \sim \epsilon$ and $\mu$ and $u$ are coupling terms generated by the elimination of $\rho_{c}$ in favor of $\zeta$. We can consider in Eqs. (1) and (4) the usual rescaling $x \longrightarrow b x^{\prime}, t \longrightarrow$ $b^{z} t^{\prime}$, and $\rho_{a} \longrightarrow b^{\delta_{a}} \rho_{a}^{\prime}$, and the rescaling of the energy field $\zeta \longrightarrow b^{\delta_{\zeta}} \zeta^{\prime}$. The rescaled coupling constants show a MF fixed point for $r=\epsilon=0$ and $z=2$. In this case the nonlinear terms couplings scale as $\mu \sim u \sim$ $b^{4-d}$. Thus, nonlinear terms are irrelevant when $d>4$, defining an upper critical dimension $d_{c}=4$ [21]. Since $r=\epsilon=0$ is the fixed point, the dissipation rate is the (temperaturelike) control parameter, with critical value $\epsilon_{c}=0$, emphasizing the role of conservation in slowly driven sandpiles. By studying Eq. (4) in the slowly driven MF stationary state $\left(\Delta \zeta=\rho_{a}=0, \rho_{c}^{\infty}=1 / g\right.$, and neglecting noise terms), we also obtain the MFT exponents for avalanche spreading: $D=4, \tau=3 / 2$, and $z=2$, in agreement with earlier analysis [7].

(ii) Fixed-energy sandpile (FES). - When $h=\epsilon=0$, the total energy $\int d^{d} x \zeta(\mathbf{x}, t)$ is conserved and plays the role of a control parameter. In this case, Eq. (1) reduces to $\partial \zeta(\mathbf{x}, t) / \partial t=\nabla^{2} \rho_{a}(\mathbf{x}, t)+\eta_{\zeta}(\mathbf{x}, t)$, where $\eta_{\zeta}(\mathbf{x}, t)$ is a conserved noise. Substituting the formal solution of this equation into Eq. (2) yields

$$
\begin{aligned}
\frac{\partial \rho_{a}(\mathbf{x}, t)}{\partial t}= & D_{a} \nabla^{2} \rho_{a}(\mathbf{x}, t)-r(\mathbf{x}) \rho_{a}(\mathbf{x}, t)-b(\mathbf{x}) \rho_{a}^{2}(\mathbf{x}, t) \\
& +w \rho_{a}(\mathbf{x}, t) \int_{0}^{t} \nabla^{2} \rho_{a}\left(\mathbf{x}, t^{\prime}\right) d t^{\prime}+\eta_{a}(\mathbf{x}, t)
\end{aligned}
$$

where we neglect higher-order terms, irrelevant by naive power-counting analysis. The coefficients $r$ and $b$ depend on position through on the initial value of $\zeta(\mathbf{x})$. Equation (5) is the Langevin equation of RFT, save the non-Markovian term and the spatial variation of $r$ and $b$; $d_{c}=4$, as in RFT. In MFT, replacing $\zeta(\mathbf{x}, 0)$ by the spatially uniform $\zeta$, we have $d \rho_{a} / d t=-\bar{r} \rho_{a}-\bar{b} \rho_{a}^{2}$, i.e., the MFT of directed percolation (DP), with critical point at $r=0$, fixing, in turn, the critical energy density $\zeta_{c}$. Close to the critical point $\left(|\Delta \zeta| / \zeta_{c} \ll 1\right), r \sim \Delta \zeta$. For $d<d_{c}$, the critical fixed point will be renormalized to $r=r^{*}$, defining a renormalized $\zeta_{c}$. Above $\zeta_{c}$, we have an active stationary state with $\rho_{a} \sim(\Delta \zeta)^{\beta}$; for $\zeta<\zeta_{c}$, the system falls into an absorbing configuration in which $\rho_{a}=0$.

Thus the FES approach to criticality $\left(\zeta \longrightarrow \zeta_{c}\right)$ is fundamentally different from the driven case $(h, \epsilon \longrightarrow 0$, followed by $h / \epsilon \longrightarrow 0)$. Note that $\zeta$ is lost as an independent parameter once $h$ and $\epsilon$ are nonzero. (Slow driving pins $\zeta$ at its critical value: if it exceeds $\zeta_{c}$, activity is generated, and thereby dissipation.) The behavior at the critical point is described by our theory with $h=\epsilon=0$ and $\zeta=\zeta_{c}$.
As in other models with infinitely many absorbing configurations, the avalanche behavior depends intimately on the initial configuration. It is also worth remarking that in the stationary driven case, the dynamics can explore only a set of recurrent configurations [22]. The FES may instead explore transient configurations that could account for the different critical behavior.

To better understand its scaling, we simulated the FES with statistically homogeneous initial conditions. We considered the BTW model with periodic boundary conditions at $(\epsilon=0, h=0)$. Initial configurations are generated by distributing at random a fixed number $N$ of particles among the $L^{d}$ sites. This defines the strictly conserved energy density $\zeta=N / L^{d}$. Once all $N$ particles have been placed, active sites topple at a unit rate with a sequential updating rule. We studied the transition from the active to the absorbing states as we varied $\zeta$. In $d=2$, using system sizes extending up to $L=1280$, we find $\zeta_{c}=2.125, \beta=$ $0.59(1), \nu=0.79(4)$, and $z=1.74(4)$ [23]. (Figures in parentheses denote uncertainties.) The corresponding DP exponents are 0.583(4), 0.73(2), and 1.76(3). Simulations of the four-dimensional model yield $\zeta_{c}=4.11(1)$ and $\beta=1.00(1)$, in good agreement with theoretical results, which predict MF values in $d \geq d_{c}=4$ (see also Fig. 2).

These results are compatible with the DP universality class, suggesting that the non-Markovian term is irrelevant, at least for homogeneous initial conditions. Preliminary results of direct integration of Eq. (5) indicate DP-compatible behavior for homogeneous initial conditions; the non-Markovian term does appear to alter the spreading exponents, as in other multiple-absorbing state models $[10,11]$. On the other hand, we find analytically that the non-Markovian term is relevant at the RFT fixed point below $d=4$, and it has to be taken into account to determine the asymptotic scaling properties. This implies

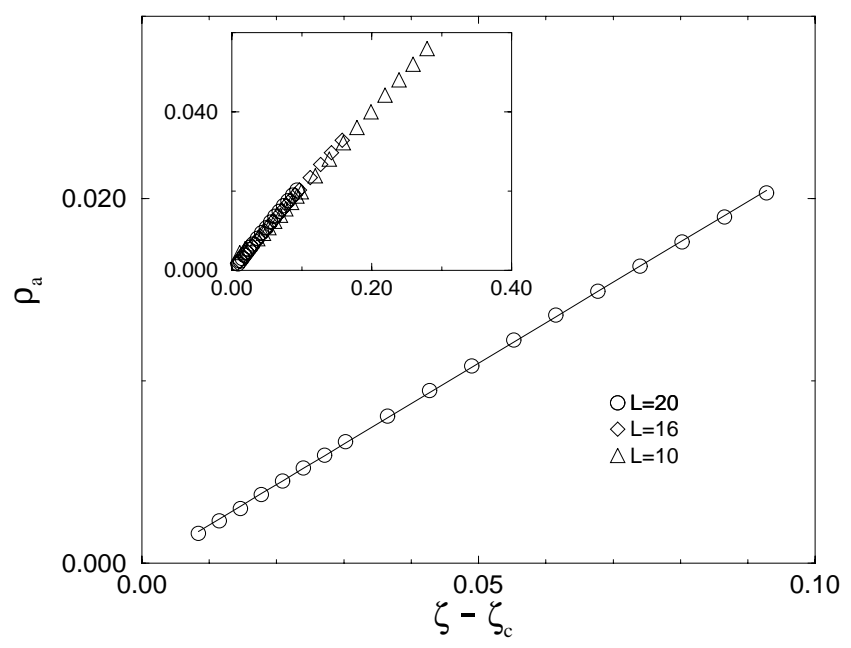

FIG. 2. Stationary active-site density as a function of $\zeta-\zeta_{c}$ for the fixed-energy BTW model in $d=4$. The inset shows $\rho_{a}$ in a larger range of $\zeta-\zeta_{c}$ and for different lattice sizes $L$. 
that to fully understand the effect of this term we need to perform a full RG perturbative expansion as well as larger numerical simulations.

In summary, our field theory elucidates the effect of driving on the critical behavior of sandpiles through their connection with other absorbing-state phase transitions [24]. Field-theoretic analysis shows that driving does not cause such a change of critical behavior in the PCP, as we have verified numerically. We believe the crucial difference is the absence of a conservation law in the PCP. Indeed, a different kind of conservation law ("local parity conservation") also changes the critical behavior of contact-process-like models [25].

Finally, we remark that our framework applies equally to the BTW and Manna sandpiles, even though the latter has a stochastic toppling rule [17]; the additional noise generates no further relevant terms. Though it remains an open question in the context of simulations [26], recent largescale numerical studies and rigorous arguments support the shared universality of the two models [27].

Our approach suggests several paths for further investigation. An open question concerns the critical behavior of fixed-energy models with nonhomogeneous initial conditions. A full renormalization group treatment of the field theory, while challenging, should yield systematic predictions for avalanche exponents. Analysis of different boundary conditions should lead to a better understanding of the scaling anomalies exhibited by sandpile models.

We thank A. Chessa, D. Dhar, L. Fabbian, P. Grassberger, E. Marinari, and $\mathrm{Y}$. Tu for comments and discussions. M.A.M., A. V., and S.Z. acknowledge partial support from the European Network Contract No. ERBFMRXCT980183. M. A.M. is partially supported from the TMR European Program No. ERBFMBIGT960925.

Note added. - During the completion of this work, we learned of large scale simulations of the BTW-FES model with parallel updating performed by Grassberger [28]. The results indicate that deviations from DP behavior persist at large system sizes, suggesting that the nonMarkovian operator in our field theory could become relevant below $d_{c}$. This is also supported by a recent theoretical approach by Lauritsen and Slava [28].

[1] T.E. Harris, The Theory of Branching Processes (Dover, New York, 1989); P. Grassberger and A. de la Torre, Ann. Phys. (N.Y.) 122, 373 (1979).

[2] R. Dickman, in Nonequilibrium Statistical Mechanics in One Dimension, edited by V. Privman (Cambridge University Press, Cambridge, 1996); J. Marro and R. Dickman, Nonequilibrium Phase Transitions (Cambridge University Press, Cambridge, 1998).

[3] W. Kinzel, Z. Phys. B 58, 229 (1985).

[4] A.L. Barabasi and H.E. Stanley, Fractal Concepts in Surface Growth (Cambridge University Press, Cambridge, 1995).
[5] P. Bak, C. Tang, and K. Wiesenfeld, Phys. Rev. Lett. 59, 381 (1987); Phys. Rev. A 38, 364 (1988).

[6] G. Grinstein, in Scale Invariance, Interfaces and Nonequilibrium Dynamics, edited by A. McKane et al., NATO ASI, Ser. B, Vol. 344 (Plenum, New York, 1995).

[7] A. Vespignani and S. Zapperi, Phys. Rev. Lett. 78, 4793 (1997); Phys. Rev. E 57, 6345 (1998).

[8] R. Dickman, A. Vespignani, and S. Zapperi, Phys. Rev. E 57, 5095 (1998).

[9] T.E. Harris, Ann. Prob. 2, 969 (1974).

[10] I. Jensen, Phys. Rev. Lett. 70, 1465 (1993); I. Jensen and R. Dickman, Phys. Rev. E 48, 1710 (1993).

[11] M. A. Muñoz, G. Grinstein, R. Dickman, and R. Livi, Phys. Rev. Lett. 76, 451 (1996); M. A. Muñoz, G. Grinstein, and R. Dickman, J. Stat. Phys. 91, 541 (1998), and references therein.

[12] C. Tang and P. Bak, Phys. Rev. Lett. 60, 2347 (1988).

[13] D. Sornette, A. Johansen, and I. Dornic, J. Phys. I (France) 5, 325 (1995).

[14] A. Chessa, E. Marinari, A. Vespignani, and S. Zapperi, Phys. Rev. E 57, R6241 (1998).

[15] S. Lübeck and K. D. Usadel, Phys. Rev. E 55, 4095 (1997); S. Lübeck, Phys. Rev. E 58, 2957 (1998).

[16] A. Díaz-Guilera, Europhys. Lett. 26, 177 (1994).

[17] S. S. Manna, J. Phys. A 24, L363 (1991).

[18] P. Grassberger, Z. Phys. B 47, 365 (1982); H. K. Janssen, Z. Phys. B 42, 151 (1981).

[19] $D_{a}$ is proportional to $\rho_{c}$, but may be treated as a constant, the neglected terms being irrelevant. The negative linear term represents sites that become stable after toppling. Setting the toppling rate to unity effectively fixes the microscopic time scale.

[20] For boundary dissipation, assuming a $d$-dimensional system of radius $R$, we have $\nabla^{2}\left\langle\rho_{a}(r)\right\rangle=-\frac{h}{D_{\zeta}}$ for $r<$ $R$, and $\left\langle\rho_{a}(r)\right\rangle=0$ at $r=R$. This implies $\left\langle\rho_{a}(r)\right\rangle \propto$ $h R^{2}\left[1-(r / R)^{2}\right]$ so that the mean activity $\bar{\rho}_{a} \sim h R^{2}$, yielding the finite-size scaling formula $\chi(R) \sim R^{\gamma / \nu}$ with $\gamma / \nu=2$.

[21] It is worth remarking that the symmetry $r \propto \epsilon$ has to be explicitly enforced if we consider a single equation, substituting in Eq. (4) the formal integration of Eq. (1). Otherwise, the spurious result $d_{c}=6$ is obtained. From the numerical point of view the value of the upper critical dimension is still a debated question $[14,15]$.

[22] D. Dhar, Phys. Rev. Lett. 64, 1613 (1990).

[23] The preliminary results reported in Ref. [8] suggesting non-DP values for $\nu$ and $z$ were in fact influenced by strong finite-size effects.

[24] This connection has been also discussed for the BakSneppen SOC model. S. Maslov, M. Paczuski, and P. Bak, Europhys. Lett. 27, 97 (1994); P. Grassberger, Phys. Lett. A 200, 277 (1995).

[25] See J.L. Cardy and U.C. Täuber, Phys. Rev. Lett. 77, 4780 (1996), and references therein.

[26] E. Milshtein, O. Biham, and S. Solomon, Phys. Rev. E 58, 303 (1998).

[27] A. Chessa, H. E. Stanley, A. Vespignani, and S. Zapperi, cond-mat/9808263; D. Dhar, cond-mat/9808047.

[28] P. Grassberger (private communication); K. B. Lauritsen and M. J. Slava (private communication). 